\title{
Special issue on machine learning-based applications and techniques in cyber intelligence
}

\author{
Lin $\mathrm{Mei}^{1} \cdot$ Zheng $\mathrm{Xu}^{2} \cdot$ Vijayan Sugumaran ${ }^{3}$
}

Published online: 1 March 2019

(c) Springer-Verlag London Ltd., part of Springer Nature 2019

The development of machine learning needs to rely on cyber intelligence technology, because it uses many theories and methods of cyber intelligence. Furthermore, it has achieved certain results. The value of machine learning as an infrastructure of cyber intelligence is about to usher in a long-term development opportunity. On the other hand, solving the problem of scalability and growth of cyber intelligence cannot be done without machine learning technology. The development of cyber intelligence also needs to rely on the environment and technology support of machine learning. It is necessary for cyber intelligence, just like human beings, to have a lot of knowledge and rich experience; behind these knowledge and experience is the need for a large number of data support. The development of cyber intelligence and machine learning will push each other to form an effective mutual promotion effect. Therefore, the development of cyber intelligence relies on machine learning [1-3], at the meanwhile; machine learning also opens a new chapter for cyber intelligence. Humans will develop intelligent technology under a new perspective of mechanism, thinking and environment of machine learning [4-6]. This special issue focuses on the theory, methods and creative solutions of cyber intelligence.

The submitted manuscripts were reviewed by experts from both academia and industry. After two rounds of reviewing, the highest quality manuscripts were accepted

Lin Mei

Linmei.trimps@hotmail.com

Zheng $\mathrm{Xu}$

xuzheng@shu.edu.cn

Vijayan Sugumaran

sugumara@oakland.edu

1 The Third Research Institute of the Ministry of Public Security, Shanghai, China

2 Shanghai University, Shanghai, China

3 Oakland University, Rochester, MI, USA for this special issue. This special issue will be published by Neural Computing and Applications as special issues. Totally, 22 papers are suggested to EiC for acceptance. The selected papers are summarized as follows:

Zhou et al. [7] study the unsupervised tensor learning problem, in which a low-rank tensor is recovered from an incomplete and grossly corrupted multi-dimensional array. Hong and $\mathrm{Yu}$ [8] summarize the relevant research achievements of collaborative filtering algorithms in recent years. By analyzing data sparsity and scalability problem in collaborative filtering algorithm, a novel collaborative filtering algorithm based on correlation coefficient (COR based) is proposed. In view of complexity and uncertainty of problems in the prediction of rockburst, a classified prediction model of rockburst using rough sets-normal cloud is established by $\mathrm{Hu}$ et al. [9]. Mohit Kumar et al. [10] design a multi-parameter online measurement method based on BP neural network algorithm. The collection, analysis, processing and display of parameters are completed through the sensing layer, the network transmission layer and the integrated application layer. A prediction model of pKa values of neutral and alkaline drugs based on particle swarm optimization algorithm and back propagation artificial neural network, called PSO-BP ANN, was established by Chen et al. [11]. Wang et al. [12] select the air quality data released in real time, obtain the historical monitoring data of air environmental pollutants and normalize the data, and then divide the sample data, and divide it into training data set and test data set in appropriate proportion. $\mathrm{Xu}$ and $\mathrm{Li}$ [13] discuss the definition of the scientific connotation of the coordinated development of regional economy and put forward three evaluation indexes for coordinated development of regional economy (the degree of regional economic integration, the degree of regional economic development gap and the speed of regional economic development). Zhang and Zhai [14] proposed a new method based on mutual information in information theory. Mutual information between signals and noises is zero theoretically; thus, random noises have 
fewer effects on first arrivals pickup with the criterion of mutual information. Ai and Yang [15] combine the particle swarm optimization algorithm to improve the support vector machine and propose a machine learning method based on particle swarm optimization support vector machine. In view of the large amount of data involved in the existing decomposition algorithm, which leads to low decomposition efficiency and high hardware requirements, a non-intrusive load decomposition method based on hidden Markov model (HMM) and improved Viterbi algorithm is proposed by Ma and Zhai [16].

In the study of Yan et al. [17], the traditional decision tree algorithm was improved, and the traditional RF method was converted into an adaptive RF (ARF) method for multi-label classification. The construction of smart cities requires the participation of nonprofit organizations, but there are still some problems in the analysis of driving factors of participation. Based on this, using the structural equation model as the research method, a public satisfaction relationship model, based on the machine learning, for nonprofit organizations participating in the construction planning of smart cities was constructed in the study of Gong et al. [18]. The Peukert equation based on temperature, current change and cycle life is established by $\mathrm{Li}$ and Bei [19] to estimate the battery capacity. Based on BP neural network theory, Cui and Jing [20] uses engineering geological database as the research and development platform. Wang et al. [21] combine the cargo floating price prediction model with the neural network algorithm (hereinafter referred to as NNA) to establish a prediction model. Zhang and Wang [22] propose a new efficiency prediction model which for the first time combines information granulation (IG) and support vector machine (SVM) with DEA model, to evaluate the future efficiency of decision making unit. In order to quantify the timevarying dependent structure between the assets and forecast the portfolio risk accurately, the difference in the preferences for asset risk is taken into consideration by Luo [23]. Pei et al. [24] proposed a novel collaborative filtering method (CBE-CF) to extract the local dense rating modules to cope with the data sparsity and the computational efficiency of the traditional recommendation algorithms, by introducing the information entropy and bi-clustering into collaborative filtering. Liu and $\mathrm{Li}$ [25] discuss the applicability of Kalman filter in 3D dynamic monitoring of environmental cost. The methods of removing invalid records, manually filling in vacancy values and using global constant to fill in vacancy values are used by Feng and Fan [26] to preprocess semantic ontology. The image feature point detection method is studied by $\mathrm{Wu}$ [27], which commonly includes image edge information-based feature detection method, corner information-based detection method and various interest operators. Based on machine learning algorithm, Ma and Lv [28] improved the machine learning algorithm and named it MLIA algorithm.

Acknowledgements The guest editors would like to thank Prof. John MacIntyre who is the editor in chief of Neural Computing and Applications. His help and trust is the most important thing for the success of this SI. The guest editors would like to thank the reviewers for their high-quality reviews, which provided insightful and constructive feedback to the authors of the papers.

\section{References}

1. Zhang S, Wei Z, Wang Y, Liao T (2018) Sentiment analysis of Chinese micro-blog text based on extended sentiment dictionary. Future Generat Comput Syst 81:395-403

2. Zhang S, Liu W, Deng X-L, Xu Z, Raymond Choo K-K (2018) Micro-blog topic recommendation based on knowledge flow and user selection. J Comput Sci 26:512-521

3. Zhang S, Zhu H, Xu Z (2017) The extraction method of new logining word/term for social media based on statistics and N-increment. J Ambient Intell Human Comput. https://doi.org/10. 1007/s12652-017-0638-6

4. Chen Y, Ding S, Xu Z, Zheng H, Yang S (2019) Blockchainbased medical records secure storage and medical service framework. J Med Syst 43(1):1-9

5. Wang X, Sugumaran V, Zhang H, Zheng X (2018) A capability assessment model for emergency management organizations. Inf Syst Front 20(4):653-667

6. Ye J, Zheng X, Ding Y (2018) Image search scheme over encrypted database. Future Generat Comp Syst 87:251-258

7. Meng Z, Zhou Y, Zhao Y (2018) Unsupervised learning low-rank tensor from incomplete and grossly corrupted data. Neural Comput Appl. https://doi.org/10.1007/s00521-018-3899-x

8. Hong B, Yu M (2018) A collaborative filtering algorithm based on correlation coefficient. Neural Comput Appl. https://doi.org/ 10.1007/s00521-018-3857-7

9. Liu R, Ye Y, Hu N et al (2018) Classified prediction model of rockburst using rough sets-normal cloud. Neural Comput Appl. https://doi.org/10.1007/s00521-018-3859-5

10. Zhang W, Kumar M, Liu J (2018) Multi-parameter online measurement IoT system based on BP neural network algorithm. Neural Comput Appl. https://doi.org/10.1007/s00521-018-3856-8

11. Chen B, Zhang H, Li M (2019) Prediction of $\mathrm{pK}(\mathrm{a})$ values of neutral and alkaline drugs with particle swarm optimization algorithm and artificial neural network. Neural Comput Appl. https://doi.org/10.1007/s00521-018-3956-5

12. Wang X, Wang B (2018) Research on prediction of environmental aerosol and PM25 based on artificial neural network. Neural Comput Appl. https://doi.org/10.1007/s00521-018-3861-y

13. Xu Y, Li A (2019) Regional economic development coordination management system based on fuzzy hierarchical statistical model. Neural Comput Appl. https://doi.org/10.1007/s00521-018-3953-8

14. Zhang QJ, Zhai MY (2019) First break of the seismic signals in oil exploration based on information theory. Neural Comput Appl. https://doi.org/10.1007/s00521-018-3955-6

15. Ai D, Yang J (2018) A machine learning approach for cost prediction analysis in environmental governance engineering. Neural Comput Appl. https://doi.org/10.1007/s00521-018-3860-z

16. Ma YJ, Zhai MY (2018) A non-intrusive load decomposition algorithm for residents. Neural Comput Appl. https://doi.org/10. 1007/s00521-018-3862-x 
17. Wu Q, Wang H, Yan X et al (2018) MapReduce-based adaptive random forest algorithm for multi-label classification. Neural Comput Appl. https://doi.org/10.1007/s00521-018-3900-8

18. Gong Z, Li X, Liu J et al (2018) Machine learning in explaining nonprofit organizations' participation: a driving factors analysis approach. Neural Comput Appl. https://doi.org/10.1007/s00521018-3858-6

19. Li B, Bei S (2018) Estimation algorithm research for lithium battery SOC in electric vehicles based on adaptive unscented Kalman filter. Neural Comput Appl. https://doi.org/10.1007/ s00521-018-3901-7

20. Cui K, Jing X (2018) Research on prediction model of geotechnical parameters based on BP neural network. Neural Comput Appl. https://doi.org/10.1007/s00521-018-3902-6

21. Guo J, Wang J, Li Q et al (2018) Construction of prediction model of neural network railway bulk cargo floating price based on random forest regression algorithm. Neural Comput Appl. https://doi.org/10.1007/s00521-018-3903-5

22. Zhang Q, Wang C (2018) DEA efficiency prediction based on IG-SVM. Neural Comput Appl. https://doi.org/10.1007/s00521018-3904-4

23. Luo X (2019) Construction of artificial neural network economic forecasting model based on the consideration of state transition diagram. Neural Comput Appl. https://doi.org/10.1007/s00521019-04038-7

24. Jiang M, Zhang Z, Jiang J et al (2019) A collaborative filtering recommendation algorithm based on information theory and biclustering. Neural Comput Appl. https://doi.org/10.1007/s00521018-3959-2

25. Liu S, Li S (2019) Three-dimensional dynamic monitoring of environmental cost based on state-space model. Neural Comput Appl. https://doi.org/10.1007/s00521-018-3960-9

26. Feng Y, Fan L (2019) Ontology semantic integration based on convolutional neural network. Neural Comput Appl. https://doi. org/10.1007/s00521-019-04043-w

27. Wu Y (2019) Research on feature point extraction and matching machine learning method based on light field imaging. Neural Comput Appl. https://doi.org/10.1007/s00521-018-3962-7

28. Ma X, Lv S (2019) Financial credit risk prediction in internet finance driven by machine learning. Neural Comput Appl. https:// doi.org/10.1007/s00521-018-3963-6

Publisher's Note Springer Nature remains neutral with regard to jurisdictional claims in published maps and institutional affiliations. 